\title{
BMJ Open How do healthcare professionals perceive physical activity prescription for community-dwelling people with COPD in Australia? A qualitative study
}

\author{
Aroub Lahham (D) ,1,2,3 Angela T Burge, ${ }^{1,2,3,4}$ Christine F McDonald, ${ }^{2,5,6}$ \\ Anne E Holland ${ }^{1,2,3,4}$
}

To cite: Lahham A, Burge AT, McDonald CF, et al. How do healthcare professionals perceive physical activity prescription for communitydwelling people with COPD in Australia? A qualitative study. BMJ Open 2020;10:e035524. doi:10.1136/ bmjopen-2019-035524

- Prepublication history and additional material for this paper are available online. To view these files, please visit the journal online (http://dx.doi. org/10.1136/bmjopen-2019035524).

Received 07 November 2019 Revised 10 February 2020 Accepted 06 July 2020

Check for updates

(C) Author(s) (or their employer(s)) 2020. Re-use permitted under CC BY-NC. No commercial re-use. See rights and permissions. Published by BMJ.

For numbered affiliations see end of article.

Correspondence to

Dr Aroub Lahham;

aroub.lahham@monash.edu

\section{ABSTRACT}

Objectives Clinical practice guidelines recommend that people with chronic obstructive pulmonary disease (COPD) should be encouraged to increase their physical activity levels. However, it is not clear how these guidelines are applied in clinical practice. This study aimed to understand the perspectives of respiratory healthcare professionals on the provision of physical activity advice to people with COPD. These perspectives may shed light on the translation of physical activity recommendations into clinical practice.

Design A qualitative study using thematic analysis. Setting Healthcare professionals who provided care for people with COPD at two major tertiary referral hospitals in Victoria, Australia.

Participants 30 respiratory healthcare professionals including 12 physicians, 10 physical therapists, 4 nurses and 4 exercise physiologists.

Interventions Semistructured voice-recorded interviews were conducted, transcribed verbatim and analysed by two independent researchers using an inductive thematic analysis approach.

Results Healthcare professionals acknowledged the importance of physical activity for people with COPD.

They were conscious of low physical activity levels among such patients; however, few specifically addressed this in consultations. Physicians described limitations including time constraints, treatment prioritisation and perceived lack of expertise; they often preferred that physical therapists provide more comprehensive assessment and advice regarding physical activity. Healthcare professionals perceived that there were few evidence-based strategies to enhance physical activity. Physical activity was poorly differentiated from the prescription of structured exercise training. Although healthcare professionals were aware of physical activity guidelines, few were able to recall specific recommendations for people with COPD.

Conclusion Practical strategies to enhance physical activity prescription may be required to encourage physical activity promotion in COPD care.

\section{BACKGROUND}

Physical activity is the strongest predictor of all-cause mortality in people with chronic obstructive pulmonary disease (COPD). ${ }^{1}$

\section{Strengths and limitations of this study}

- This study is the first to elicit the perspectives of respiratory healthcare professionals working in tertiary care on providing physical activity advice to people with chronic obstructive pulmonary disease.

- This study comprises a focused representation on the perspectives of respiratory specialists and cardiopulmonary physical therapists.

- Thematic analysis was utilised for data synthesis in this study because its results can be transparently communicated to healthcare practitioners, policymakers and the general population.

- Independent data syntheses by two investigators and routine group discussions improved the validity of our results and credibility of data synthesis.

- The interviews took place in two Australian hospitals in one city, this narrow representation limits the generalisability of the reported findings on clinical practice worldwide.

Participating in sufficient levels of physical activity is associated with reduced diseaserelated hospitalisations, improved breathlessness and enhanced health-related quality of life. ${ }^{23}$ Despite the strong association between higher physical activity levels and enhanced disease outcomes, people with COPD are highly inactive. ${ }^{4}$ Indeed, people with COPD spend less than half the time in moderate to vigorous intensity physical activity (MVPA) than do healthy age-matched controls. ${ }^{5}$

'Physical activity' and 'exercise training' are related terms related but with different concepts. While physical activity is defined as any bodily movement produced by skeletal muscles and requiring energy expenditure, ${ }^{6}$ exercise training is a subset of physical activity that is structured, planned and repetitive. ${ }^{6}$ Thus physical activity could include both time spent in incidental movement during daily life, as well as structured exercise programmes such as pulmonary rehabilitation. 
International public health guidelines recommend that older adults and adults with chronic illness participate in at least 150 min of MVPA per week within their capabilities. ${ }^{78}$ The Global Initiative for Chronic Obstructive Lung Disease recommends that physical activity is advised for all patients regardless of disease severity. ${ }^{9}$ However, it is not known whether healthcare professionals apply these guidelines to prescribe physical activity for people with COPD. The aim of this study was to elicit COPD healthcare professionals' perspectives on the importance and prescription of physical activity in clinical practice.

\section{METHODS}

\section{Design}

The interview schedule was devised based on the systematic yet flexible principles of grounded theory to allow for explorative and in-depth discussions around physical activity prescription for people with COPD. ${ }^{10}$ Semistructured and open-ended questions were designed by two female PhD-qualified physical therapist researchers, one with 10 years experience in designing qualitative research including formal training and is an expert in pulmonary rehabilitation research in chronic lung disease (AEH). AEH provided mentorship to the second researcher (AL) who had a 5 years experience in conducting qualitative research and 7 years experience in conducting research around physical activity promotion and exercise training for people with COPD (AL).

Interviews were designed to last for approximately 20 min. Healthcare professionals were asked about their perceptions of the importance of physical activity for people with COPD, their approach to eliciting information from patients about their activity levels and their views on physical activity prescription in clinical practice. A detailed list of interview questions is presented in box 1. Interview schedule was piloted, and order, wording and relevance of the questions were reviewed prior to the commencement of the study. Ethics approval was obtained from La Trobe University Human Ethics, Alfred Health Human Ethics and Austin Health Human Ethics Committees.

\section{Setting}

Participants were healthcare professionals who provided care for people with COPD at the Alfred and Austin Hospitals in Melbourne, Australia. Participants included respiratory physicians, respiratory physical therapists, COPD nurses and exercise physiologists.

\section{Procedure}

Potential participants were informed about the study by email invitations from administrative offices. Willing participants contacted the second researcher (AL) to organise the time and place for the interview. Written informed consent was obtained from all participants. All the interviews were conducted by one researcher (AL).

\section{List of interview questions}

1. Could you tell me your thoughts about the role of physical activity for patients with COPD?

2. When you are treating people with COPD, how do you approach the topic of physical activity?

If it did not come up:

- Could you tell me about any particular activities you recommend?

- How do you decide how much physical activity someone with COPD should do?

3. Could you tell me about any ways in which your approach differs according to the severity of COPD?

4. Could you tell me about the relative roles of pulmonary rehabilitation and physical activity for COPD?

5. Who do you think should prescribe physical activity for patients with COPD?

6. Can you think about a situation of which prescribing physical activity for patients with COPD is not a priority?

7. Can you think of anything that would make it easier for healthcare professionals to prescribe physical activity for patients with COPD?

8. What is your understanding of what clinical guidelines say about physical activity for COPD?

COPD, chronic obstructive pulmonary disease.

\section{Data analysis}

Voice-recorded interviews were transcribed verbatim and deidentified. Two independent researchers (AL and ATB) analysed the transcriptions using an iterative inductive thematic analysis approach. ${ }^{11}$ According to the principles of inductive thematic analysis, a pre-existing coding framework was not set, and data were approached free of researchers' theoretical interests in physical activity prescription. ${ }^{11}$ In order to gain an immediate impression of data content, familiarisation by reading and rereading transcripts first took place. Preliminary descriptive coding was then carried out and progressed to patterned coding. Patterned codes were then combined to subthemes and overarching themes. ${ }^{10}$ The independent researchers then met to discuss themes. Any disagreements were resolved by a third independent researcher $(\mathrm{AEH})$. All three investigators who were involved in the data analysis process are female physical therapist researchers. Investigator triangulation was employed by involving three investigators in the analysis process.

Routine in-depth discussions regarding themes and data content took place between the two independent researchers. Data analysis was conducted concurrently with data collection. Data saturation was considered to have been achieved when two independent researchers agreed that no new themes emerged from analysis of the transcripts. ${ }^{12}$ The three researchers involved in the analysis commented on the themes after finalising the data analysis.

\section{Patient and public involvement}

The patients and the public were not involved in the design or planning of the study. 
Table 1 Themes derived from the interviews

\begin{tabular}{|c|c|}
\hline Major themes & Subthemes \\
\hline $\begin{array}{l}\text { Theme } 1 . \text { Physical activity is important, however people } \\
\text { with COPD are inactive }\end{array}$ & $\begin{array}{l}\text { Physical activity is a key component of disease management for } \\
\text { people with COPD. } \\
\text { People with COPD are inactive. } \\
\text { Patient education about physical activity is critical. }\end{array}$ \\
\hline $\begin{array}{l}\text { Theme } 2 \text {. Assessment of physical activity in people } \\
\text { with COPD is challenging }\end{array}$ & $\begin{array}{l}\text { Difficulties in assessing physical activity. } \\
\text { Healthcare professionals commonly inquire about functional } \\
\text { capacity. } \\
\text { Exertional dyspnoea is perceived as an important indicator of } \\
\text { activity levels. }\end{array}$ \\
\hline $\begin{array}{l}\text { Theme } 3 \text {. Physical activity advice is not a priority and } \\
\text { healthcare professionals may lack expertise }\end{array}$ & $\begin{array}{l}\text { Physical activity advice should be individualised for each patient. } \\
\text { Lack of perceived expertise when providing activity advice. } \\
\text { Physical activity advice is not always prioritised. }\end{array}$ \\
\hline $\begin{array}{l}\text { Theme } 4 \text {. Promotion of physical activity for people with } \\
\text { COPD is a shared responsibility }\end{array}$ & $\begin{array}{l}\text { Activity promotion should occur in every medical interaction. } \\
\text { Healthcare professionals are uncertain about the differences } \\
\text { between physical activity and exercise } \\
\text { The general practitioner role in prescribing physical activity is } \\
\text { crucial. } \\
\text { Healthcare professionals need more education about physical } \\
\text { activity prescription. }\end{array}$ \\
\hline $\begin{array}{l}\text { Theme } 5 \text {. Effective strategies to improve participation in } \\
\text { physical activity are needed }\end{array}$ & $\begin{array}{l}\text { Few effective interventions to improve physical activity are } \\
\text { available. } \\
\text { Pulmonary rehabilitation improves physical activity. } \\
\text { Not all patients are referred to pulmonary rehabilitation. }\end{array}$ \\
\hline $\begin{array}{l}\text { Theme } 6 \text {. Physical activity recommendations are poorly } \\
\text { recalled }\end{array}$ & $\begin{array}{l}\text { Physical activity guidelines are poorly recalled. } \\
\text { Few drivers to translate activity guidelines into daily clinical } \\
\text { practice. }\end{array}$ \\
\hline
\end{tabular}

COPD, chronic obstructive pulmonary disease.

\section{RESULTS}

Thirty-three healthcare professionals replied to the email invitations; three physicians were subsequently unavailable. Those interviewed included 12 physicians, 10 physical therapists, 4 nurses and 4 exercise physiologists. Of the interviewed healthcare professionals, eight worked in specialist outpatient clinics, eight in inpatients ward settings, nine in pulmonary rehabilitation programmes and five in community outreach programmes. Major themes and subthemes are listed in table 1. Supporting quotations for themes and subthemes are available online (online supplementary table S1).

\section{Theme 1. Physical activity is important, however people with COPD are inactive}

Participating in regular physical activity was identified as important for maintenance of physical and mental function. Indeed, healthcare professionals described physical activity as a key component of disease management for patients with COPD.

(Physical activity) is a key and... scientifically proven component of their management. C01, Physician

(Physical activity) also helps with how they are feeling in (terms of) well-being, because patients will feel that they are doing something and they are able to participate in activities they like...a lot of patients become a bit depressed when they become really just sitting around doing nothing. C13, Physician

(Physical activity) maintains function and encourages mobility. C07, Physician

Despite the perceived benefits of physical activity, healthcare professionals identified many challenges to participation. Fear of dyspnoea was consistently nominated as a key catalyst in the vicious cycle of physical inactivity in people with COPD.

Patients are a bit reluctant... (if) they are breathless, then they... stop pushing it and feel insecure not knowing what they need to do.

C06, Physician

The detrimental impact of inactivity on quality of life, symptoms and disease progression over time were shared concerns. The importance of education regarding physical activity for people with COPD, particularly the need to integrate education into everyday life, was frequently discussed.

It's important that patients understand that (physical activity)...is not just a part of a treatment when they first come to see a medical person; they learn to integrate (it) into their whole life... .C07, Physical therapist 
Theme 2. Assessment of physical activity in people with COPD is challenging

Physical activity was generally considered as difficult to assess and therefore was infrequently monitored in clinical practice.

Reduction in physical activity is an important symptom... (but) it is not something that people obviously complain about... and from a clinician's point of view it can be difficult to identify. C05, Physician

Discussions about participation in physical activity were usually initiated with general enquiries regarding daily routines and capacity for functional activities in everyday life, especially walking.

I ask what they are able to do, in terms of physical activity...how far they can walk and if they can't walk that far, I might ask them how do they cope with other activities such as dressing, you know, showering and those sorts of things. C01, Physician

A shared perception across professions was that participation in physical activity was not necessarily reflected by traditional assessments of disease severity.

That is something that needs to be decided on an individual basis...for that particular patient. You can't use $\mathrm{FEV}_{1}$ (Forced Expiratory Volume in one second) to decide. C05, Physician

Enquiring about activities that provoke dyspnoea was commonly nominated as the main assessment strategy for physical activity.

I usually ask them what are the things that they do that made them most breathless and how do they manage that. C04, Nurse

\section{Theme 3. Physical activity advice is not a priority and healthcare professionals may lack expertise}

Healthcare professionals stated their readiness to address physical activity in everyday practice. They acknowledged that to be meaningful, physical activity advice should be individualised to the person's preferences, capabilities and daily routines.

(Healthcare professionals) say 'Just go for a walk around the shops' or 'Just get out of bed and walk around the block' and often it is not enough...it needs to be more individualised and structured. C02, Physical therapist

Despite this awareness, advice regarding increasing physical activity was not prioritised in clinical practice, particularly in the context of competing treatment demands and time constraints.

I don't (talk about physical activity)! Unfortunately, to date, a lot of my practice, and I think a lot of physicians' focus, is around medication issues. C02, Physician
Most medical and nursing healthcare professionals perceived limitations to their expertise in recommending physical activity.

The problem is...what are the right physical activities to be performing?...the frequency?...it is difficult, as a medical practitioner...to have the knowledge of what they can do. It is a little bit difficult to go into that with great detail with patients. C02, Physician

Theme 4. Promotion of physical activity for people with COPD is a shared responsibility

There was consensus that the promotion of physical activity was an obligation shared by all members of the healthcare team. Every clinical interaction was perceived as an opportunity for providing advice on physical activity.

Someone has got to be ready to have the conversation...if you're not going to see them again, you can encourage them, put the idea in their head get them to understand the importance of physical activity as a priority. Give them the information about how it may be beneficial for them, and at least that's an opening... .

\section{C11, Physical therapist}

However, prescription of such physical activity was perceived by most physicians and nurses as being the domain of the physical therapist. This perception was justified by highlighting the physical therapists' detailed knowledge and experience in exercise prescription.

To be done properly, it should be done by a physio. It's best that someone actually sees them... (and) takes appropriate history...we are not trained for that C07, Physician

However, when asked about their views on the role of physical activity in routine practice, physical therapists explained that, unlike exercise training, physical activity should be prescribed by all healthcare professionals. They further elaborated that while exercise prescription involves highly structured activities with intensity and duration determined by personalised functional assessments, daily physical activity could be encouraged by all members of the healthcare team.

If patients need specific advice on exercise prescription...if they have comorbidities or something that's going to make exercise prescription a little bit more complex...ideally...they should be referred on to a physio (physical therapist) or an exercise physiologist... ...I don't think exercise prescription is everyone's job...(but) physical activity is everyone's job. C11, Physical therapist

The crucial role of general practitioners (GPs) in the management of COPD was highlighted, but the challenges faced in primary care (including time constraints and perceived lack of expertise) were also acknowledged. 
There is a proportion of (people with) COPD that never get to see the lung specialist. They are only being seen by the GP and that...is difficult because different GPs...may not be as comfortable...to get the patient to do some (physical activity). C08, Physician

As a result, healthcare professionals strongly highlighted the importance of education regarding prescription and importance of physical activity.

Better knowledge among healthcare professionals is important...some people don't understand that reduced physical activity is an issue...they don't often know the evidence. C05, Physician

\section{Theme 5. Effective strategies to improve participation in physical activity are needed}

Healthcare professionals highlighted the lack of evidencebased interventions to improve activity in people with COPD. Pulmonary rehabilitation was perceived as an effective strategy to improve physical activity.

I don't have lots of recommendations... any real dayto-day suggestions...any practical solutions...other than... refer them to pulmonary rehabilitation. C05, Physician

The majority of healthcare professionals described referral to pulmonary rehabilitation to improve physical activity across the disease spectrum, in addition to expressing their confidence in the programmes' ability to reduce symptoms of COPD and improve quality of life.

(Physical activity) is something that I don't necessarily focus on that much because I send them all to pulmonary rehabilitation and let them take care of it. C12, Physician

However, referral to pulmonary rehabilitation was not always part of clinical practice.

(If) they say no (to referral for pulmonary rehabilitation), I say 'That's fine. It's just what we recommend'.

\section{C12, Physician}

In general, healthcare professionals acknowledged the need for more evidence-based strategies that promote a physically active lifestyle for patients with COPD.

We've got to think of more innovative ways of engaging with our patients and working with them...there will always be that group of patients who would not go to a pulmonary rehab programme. C04, Nurse

\section{Theme 6. Physical activity recommendations are poorly recalled}

Some healthcare professionals were aware of guidelines regarding physical activity; however, only few were able to recall specific recommendations.

I don't know of any guidelines per se for just general physical activity. There may be some; I am just not aware of any. C01, Physician
Others acknowledged the importance of developing practical strategies necessary to facilitate the translation of guidelines into the model of care for COPD.

I have no doubt that they exist in the guidelines... (which are) a great resource... (but) tend to be long and detailed and (not) easily...incorporated on the spot into clinical practice C03, Physician

\section{DISCUSSION}

This qualitative interview-based study assessed the perspectives of healthcare professionals regarding the provision of physical activity advice to people with COPD. Respiratory healthcare professionals perceived physical activity as a key component of disease management and felt responsible for promoting the importance of physical activity during routine care. Highlighted barriers to prescription were limited time, lack of perceived expertise and prioritisation of other treatments during consultations. In addition, healthcare professionals highlighted the lack of robust evidence regarding effective strategies to promote physical activity in people with COPD. They also felt uncertain regarding the provision of physical activity recommendations for patients with COPD. This report suggests the need for practical strategies that facilitate translation of physical activity recommendation into clinical practice.

Our analysis showed that healthcare professionals perceive physical activity advice as an important component of disease management and should occur routinely in clinical practice. Consistently, several studies reported that older adults who received advice about their physical activity levels during their consultations participated in more activities than those who did not. ${ }^{13-15}$ However, most of these reports were in the primary care setting and there are few randomised controlled trials. To date, evidence on the true effects of physical activity advice provision as an important component of COPD care are lacking.

Despite the perceived importance of physical activity advice, it was clear that many health professionals were ambivalent about delivering this in their routine practice. Perceived lack of expertise, time constraints and uncertainty regarding effective strategies were highlighted as barriers to implementation. They also expressed the need to improve their knowledge regarding the content and appropriateness of the physical activity advice provided in clinical practice. This was supported by an Australian interview-based qualitative study exploring barriers to translating physical activity guidelines into practice in patients with lung cancer. ${ }^{16}$ In that study, factors hindering promotion were perceived to relate to healthcare professionals' lack of knowledge and the absence of standardised pathways that facilitate prescription in clinical practice. ${ }^{16}$ The study highlighted the importance of enhancing healthcare professionals' knowledge and confidence in providing advice on physical activity, 
especially that of GPs. ${ }^{16}$ It appears that difficulties with implementing physical activity advice in clinical practice are common and are applicable to people with many chronic conditions. ${ }^{17} 18$ While it is encouraging that health professionals in our study considered physical activity to be important for people with COPD, our results suggest that further support and education is required for physical activity prescription to become routine practice.

Healthcare professionals perceived the lack of robust evidence on effective physical activity interventions for people with COPD as an important barrier to implementation. ${ }^{19}$ A summary of recent evidence suggested that exercise training and physical activity counselling are effective in producing small but significant effects on physical activity levels in people with COPD. ${ }^{20}$ While pulmonary rehabilitation is strongly recommended to improve exercise capacity and quality of life, its impact on physical activity levels is not well established. ${ }^{21}$ In addition, many people with COPD find pulmonary rehabilitation programmes difficult to access and uptake is poor. ${ }^{22}$ It may be easier for some patients to engage in simple strategies to increase physical activity in daily life, rather than attend a formal programme. High-quality research providing robust evidence about successful strategies to encourage physical activity in daily life may increase healthcare professionals' confidence in advocating activity participation for patients with COPD. However, reaching consensus regarding effective interventions does not interfere with the urgent need to prompt professionals to routinely advise about physical activity during their consultations.

Healthcare professionals conveyed contrasting views on the role of physical activity prescription in clinical practice in this study, with physical therapists viewing things differently from others. While there was a belief among most physicians and nurses that prescription of activity is the domain of the physical therapist due to their expertise in exercise prescription, physical therapists described that exercise training as being different from participation in physical activity which could be encouraged using practical strategies acquired by the whole healthcare team. ${ }^{6}$ It appears that physicians viewed physical activity prescription to be limited to exercise training prescription; an important element of pulmonary rehabilitation programmes. Physical therapists also averred that education of healthcare professionals about the relative roles of physical activity and exercise is vital when treating patients in clinical practice.

An example of a practical model to promote physical activity prescription was recently published. The American Associations of Nurse Practitioners issued an evidence-based framework for nurses to use in clinical practice in order to provide physical activity prescription for people with COPD and asthma. ${ }^{23}$ The approach appeared promising for upskilling nurses to address physical activity prescription with their patients. ${ }^{23}$ Perhaps the development of a standardised physical activity framework guided by international recommendations could provide an effective approach that would facilitate physical activity prescription by a range of healthcare professionals. It is possible that, when brief and timely physical activity advice to patients is repeated in every healthcare encounter, meaningful behaviour change could occur in this sedentary population.

\section{Strength and limitations}

This is the first study to report the perspectives of Australian respiratory healthcare professionals on providing physical activity advice to people with COPD. One of the limitations of this study was the small number of COPD nurses and exercise physiologists who were interviewed which might have influenced the results. Our participants had volunteered to participate in a study about physical activity, and it is possible that our participants were not representative of the broader group of health professionals involved in COPD care. Additionally, we did not report information about participants physical activity behaviour and demographics which may have influenced the reported perspectives regarding physical activity prescription. Due to the interviewees limited time and busy clinical workload, themes were not sent back to the interviewees for further revisions. As the interviews took place in two Australian hospitals in one city, this narrow representation might not be reflective of clinical practice worldwide. However, a strength of this study is the significant representation of views from specialist respiratory physicians and respiratory physical therapists caring for patients with COPD.

\section{CONCLUSION}

Healthcare professionals perceive physical activity promotion as an important component of care that should be provided for people with COPD. Subjective assessment of physical activity levels is routinely undertaken by healthcare professionals; however, physical activity advice and prescription are not always prioritised in routine practice. This report emphasises the need for healthcare professionals to acquire strategies for physical activity prescription in practice, and the need for future research to find effective interventions that can be readily implemented to increase physical activity in people with COPD.

\section{Author affiliations \\ ${ }^{1}$ Discipline of Physiotherapy, La Trobe University, Melbourne, Victoria, Australia ${ }^{2}$ Institute for Breathing and Sleep, Melbourne, Victoria, Australia \\ ${ }^{3}$ Department of Allergy, Immunology and Respiratory Medicine, Monash University, Melbourne, Victoria, Australia \\ ${ }^{4}$ Department of Physiotherapy, Alfred Hospital, Melbourne, Victoria, Australia ${ }^{5}$ Respiratory and Sleep Medicine, Austin Health, Heidelberg, Victoria, Australia ${ }^{6}$ Department of Medicine, The University of Melbourne, Melbourne, Victoria, Australia}

Contributors AL, CFM and AEH helped in study concept and design. Ethics approval and data acquisition were obtained by AL. Data analysis was done by AL, ATB and AEH. AL drafted the manuscript. Critical revision of the manuscript was performed by all the authors. All authors gave final approval of the manuscript.

Funding The authors have not declared a specific grant for this research from any funding agency in the public, commercial or not-for-profit sectors. 
Competing interests None declared.

Patient consent for publication Not required.

Ethics approval Ethics approval was obtained from La Trobe University Human Ethics Committee, Alfred Health Human Ethics Committee (380/14) and Austin Health Human Ethics Committee (LNR/14/Austin/629). Written informed consent was obtained from all study participants.

Provenance and peer review Not commissioned; externally peer reviewed.

Data availability statement № additional data are available.

Open access This is an open access article distributed in accordance with the Creative Commons Attribution Non Commercial (CC BY-NC 4.0) license, which permits others to distribute, remix, adapt, build upon this work non-commercially, and license their derivative works on different terms, provided the original work is properly cited, appropriate credit is given, any changes made indicated, and the use is non-commercial. See: http://creativecommons.org/licenses/by-nc/4.0/.

ORCID iD

Aroub Lahham http://orcid.org/0000-0003-2090-0746

\section{REFERENCES}

1 Waschki B, Kirsten A, Holz O, et al. Physical activity is the strongest predictor of all-cause mortality in patients with COPD: a prospective cohort study. Chest 2011;140:331-42.

2 Esteban C, Arostegui I, Aburto M, et al. Influence of changes in physical activity on frequency of hospitalization in chronic obstructive pulmonary disease. Respirology 2014;19:330-8.

3 Esteban C, Quintana JM, Aburto M, et al. Impact of changes in physical activity on health-related quality of life among patients with COPD. Eur Respir J 2010;36:292-300.

4 Pitta F, Troosters T, Spruit MA, et al. Characteristics of physical activities in daily life in chronic obstructive pulmonary disease. $\mathrm{Am} J$ Respir Crit Care Med 2005;171:972-7.

5 Troosters T, Sciurba F, Battaglia S, et al. Physical inactivity in patients with COPD, a controlled multi-center pilot-study. Respir Med 2010;104:1005-11.

6 Caspersen CJ, Powell KE, Christenson GM. Physical activity, exercise, and physical fitness: definitions and distinctions for healthrelated research. Public Health Rep 1985;100:126.

7 Nelson ME, Rejeski WJ, Blair SN, et al. Physical activity and public health in older adults: recommendation from the American College of sports medicine and the American heart association. Circulation 2007:116:1094.

8 Smoking, nutrition, alcohol, physical activity (SNAP). A population health guide to behavioural risk factors in general practice. Royal Australian College of general practitioners. 2nd edn, 2015.
9 The global strategy for the diagnosis MaPoC, global initiative for chronic obstructive lung disease (gold), 2017. Available: http:// goldcopd.org

10 Lingard L, Albert M, Levinson W. Grounded theory, mixed methods, and action research. BMJ 2008;337:a567.

11 Braun V, Clarke V. Using thematic analysis in psychology. Qual Res Psychol 2006;3:77-101.

12 O’Reilly M, Parker N. 'Unsatisfactory Saturation': a critical exploration of the notion of saturated sample sizes in qualitative research. Qual Res 2013;13:190-7.

13 Noordman J, Verhaak P, van Dulmen S. Discussing patient's lifestyle choices in the consulting room: analysis of GP-patient consultations between 1975 and 2008. BMC Fam Pract 2010;11:87.

14 Grandes G, Sanchez A, Sanchez-Pinilla RO, et al. Effectiveness of physical activity advice and prescription by physicians in routine primary care: a cluster randomized trial. Arch Intern Med 2009;169:694-701.

15 Hirvensalo M, Heikkinen E, Lintunen T, et al. The effect of advice by health care professionals on increasing physical activity of older people. Scand J Med Sci Sports 2003;13:231-6.

16 Granger CL, Denehy L, Remedios L, et al. Barriers to translation of physical activity into the lung cancer model of care. A qualitative study of clinicians' perspectives. Ann Am Thorac Soc 2016;13:2215-22.

17 Avery L, Flynn D, Dombrowski SU, et al. Successful behavioural strategies to increase physical activity and improve glucose control in adults with Type 2 diabetes. Diabet Med 2015;32:1058-62.

18 Berra K, Rippe J, Manson JE. Making physical activity counseling a priority in clinical practice: the time for action is now. JAMA 2015;314:2617-8.

19 Burge AT, Cox NS, Abramson MJ, et al. Interventions for promoting physical activity in people with COPD. Cochrane Database Syst Rev 2017;5.

20 Lahham A, McDonald CF, Holland AE. Exercise training alone or with the addition of activity counseling improves physical activity levels in COPD: a systematic review and meta-analysis of randomized controlled trials. Int $\mathrm{J}$ Chron Obstruct Pulmon Dis 2016;11:3121-36

21 Cox NS, Oliveira CC, Lahham A, et al. Pulmonary rehabilitation referral and participation are commonly influenced by environment, knowledge, and beliefs about consequences: a systematic review using the theoretical domains framework. J Physiother 2017;63:84-93

22 Keating A, Lee AL, Holland AE. Lack of perceived benefit and inadequate transport influence uptake and completion of pulmonary rehabilitation in people with chronic obstructive pulmonary disease: a qualitative study. J Physiother 2011;57:183-90.

23 Corbridge SJ, Nyenhuis SM. Promoting physical activity and exercise in patients with asthma and chronic obstructive pulmonary disease. $J$ Nurse Pract 2017;13:41-6. 